\title{
Examination of the antimalarial potential of experimental aminoquinolines: poor in vitro effect does not preclude in vivo efficacy
}

\author{
Jelena Srbljanović a , Tijana Štajner ${ }^{a}$, Jelena Konstantinović ${ }^{b}$, Nataša Terzić-Jovanovićc ${ }^{\text {, }}$ \\ Aleksandra Uzelac a , Branko Bobić ${ }^{\mathrm{a}}$, Bogdan A. Šolaja ${ }^{\mathrm{b}, \mathrm{d}}$, Olgica Djurković-Djaković a,* \\ a Institute for Medical Research, University of Belgrade, Dr Subotića 4, P.O. Box 39, 11129 Belgrade 102, Serbia \\ ${ }^{\mathrm{b}}$ Faculty of Chemistry, University of Belgrade, Studentski trg 16, P.O. Box 51, 11158 Belgrade, Serbia \\ ${ }^{c}$ Institute of Chemistry, Technology and Metallurgy, 11000 Belgrade, Serbia \\ d Serbian Academy of Sciences and Arts, Knez Mihailova 35, 11000 Belgrade, Serbia
}

\section{A R T I C L E I N F O}

\section{Article history:}

Received 27 March 2017

Accepted 10 June 2017

\section{Keywords:}

Malaria

Aminoquinolines

LDH assay

Thompson test

Adamantane

\begin{abstract}
A B S T R A C T
Malaria remains a major disease in the developing world and globally is the most important parasitic disease causing significant morbidity and mortality. Because of widespread resistance to conventional antimalarials, including chloroquine (CQ), new drugs are urgently needed. Here we report on the antimalarial efficacy, both in vitro and in vivo, of a series of aminoquinoline derivatives with adamantane or benzothiophene as a carrier. In vitro efficacy was evaluated by a lactate dehydrogenase (LDH) assay in cultures of a CQ-sensitive (3D7) and CQ-resistant (Dd2) strain of Plasmodium falciparum. Of a series of 26 screened compounds, 12 that exerted a growth inhibition rate of $\geq 50 \%$ were further examined in vitro to determine the $50 \%$ inhibitory concentration $\left(\mathrm{IC}_{50}\right)$ values. Nine compounds shown in preliminary experiments to be non-toxic in vivo were evaluated in C57BL/6 mice infected with Plasmodium berghei ANKA strain using a modified Thompson test. All nine compounds examined in vivo prolonged the survival of treated versus untreated mice, four of which afforded $\geq 60 \%$ survival. Most notably, two of these compounds, both with the adamantane carrier, afforded complete cure ( $100 \%$ survival and parasite clearance). Interestingly, one of these compounds had no in vitro effect against the CQ-resistant $P$. falciparum strain. Better in vivo compared with in vitro results suggest a role for compound metabolites rather than the compounds themselves. The results presented here point to adamantane as a carrier that enhances the antimalarial potential of aminoquinolines.
\end{abstract}

(c) 2017 Elsevier B.V. and International Society of Chemotherapy. All rights reserved.

\section{Introduction}

Malaria, caused by protozoan parasites of the Plasmodium genus, continues to be a major health problem in the developing world and is globally the most important parasitic disease causing significant morbidity and mortality. Human infections are caused by five species of the genus, namely Plasmodium falciparum, Plasmodium vivax, Plasmodium ovale, Plasmodium malariae and Plasmodium knowlesi. Infection resulting from the bite of an infected female Anopheles mosquito is characterised by blood and liver stages [1].

\footnotetext{
The results of this study were presented in part at the 12th European Multicolloquium of Parasitology (EMOPXII) Conference, 20-24 July 2016, Turku, Finland.

* Corresponding author. Institute for Medical Research, University of Belgrade, Dr Subotića 4, P.O. Box 39, 11129 Belgrade 102, Serbia.

E-mail address: olgicadj@imi.bg.ac.rs (O. Djurković-Djaković).
}

The World Health Organization (WHO) estimated 214 million cases of malaria and 438000 deaths in 2015 [2], with most of the deaths caused by P. falciparum. One-half of the global human population, residing in tropical and subtropical areas, is estimated to be at a risk of infection, but even the other half is facing an increasing number of imported cases, resulting in deaths and health system burden in non-endemic countries and occasional secondary transmission in areas where malaria has long ago been eradicated [3].

The efficacy of the main conventional antimalarials, including chloroquine (CQ) and artemisinin, is hampered by widespread drug resistance. Coupled with the lack of an effective vaccine, this strongly emphasises the urgent need for novel compounds to treat and prevent malaria $[4,5]$.

The mechanism of action of CQ, like all quinolones, involves activity against the erythrocyte forms of all Plasmodium spp. by preventing polymerisation of heme through its selective accumulation in the parasite food vacuole (FV). CQ forms stable complexes with heme and its removal from the FV is prevented by protonation 
Table 1

Investigated compounds grouped according to modifications at the aminoquinoline moiety.

\begin{tabular}{|c|c|c|c|}
\hline Group & Compound & Acronym & Compound no. in [19] \\
\hline \multirow[t]{3}{*}{$\mathrm{AQ}$} & $N^{1}$-(1-adamantylmethyl)- $N^{3}$-quinolin-4-ylbutane-1,3-diamine, $\mathrm{C}_{24} \mathrm{H}_{33} \mathrm{~N}_{3}$ & AQ1 & 24 \\
\hline & $N^{1}$-[2-(1-adamantyl)ethyl]- $N^{3}$-quinolin-4-ylbutane-1,3-diamine & AQ2 & 44 \\
\hline & $N$-(1-adamantylmethyl)- $N$-methyl- $N$ '-quinolin-4-ylpropane-1,3-diamine & AQ3 & Not previously published \\
\hline \multirow[t]{6}{*}{ ClAQ } & $N^{1}$-(1-adamantylmethyl)- $N^{3}$-(7-chloroquinolin-4-yl)butane-1,3-diamine & ClAQ1 & 23 \\
\hline & $N^{2}$-(1-adamantylmethyl)- $N^{1}$-(7-chloroquinolin-4-yl)propane-1,2-diamine & ClAQ2 & 10 \\
\hline & $N$-(7-chloroquinolin-4-yl)-N'-[(5-fluoro-1-benzothiophen-3-yl)methyl]propane-1,3-diamine & ClAQ3 & 58 \\
\hline & $N$-(7-chloroquinolin-4-yl)- $N^{\prime}$-[(5-fluoro-1-benzothiophen-3-yl)methyl]butane-1,4-diamine & ClAQ4 & 63 \\
\hline & $N$-(7-chloroquinolin-4-yl)- $N$ '-[(6-fluoro-1-benzothiophen-3-yl)methyl]propane-1,3-diamine & ClAQ5 & 59 \\
\hline & $N^{1}$-[2-(1-adamantyl)ethyl]- $N^{3}$-(7-chloroquinolin-4-yl)butane-1,3-diamine & ClAQ6 & 36 \\
\hline \multirow[t]{2}{*}{ FAQ } & $N^{1}$-(1-adamantylmethyl)- $N^{3}$-(3-fluoroquinolin-4-yl)butane-1,3-diamine & FAQ1 & 26 \\
\hline & $N^{1}$-[2-(1-adamantyl)ethyl]- $N^{3}$-(3-fluoroquinolin-4-yl)butane-1,3-diamine & FAQ2 & 39 \\
\hline \multirow[t]{8}{*}{ FClAQ } & $N^{1}$-(1-adamantylmethyl)- $N^{3}$-(7-chloro-3-fluoroquinolin-4-yl)butane-1,3-diamine & FClAQ1 & 25 \\
\hline & $N^{4}$-(7-chloro-3-fluoroquinolin-4-yl)- $N^{1}, N^{1}$-diethylpentane-1,4-diamine & FClAQ2 & 74 \\
\hline & $N^{1}$-(1-adamantylmethyl)- $N^{2}$-(7-chloro-3-fluoroquinolin-4-yl)propane-1,2-diamine & FClAQ3 & 20 \\
\hline & $N^{2}$-(1-adamantylmethyl)- $N^{1}$-(7-chloro-3-fluoroquinolin-4-yl)propane-1,2-diamine & FClAQ4 & 21 \\
\hline & $N^{1}$-[2-(1-adamantyl)ethyl]- $N^{3}$-(7-chloro-3-fluoroquinolin-4-yl)butane-1,3-diamine & FClAQ5 & 38 \\
\hline & $N^{1}$-(1-adamantylmethyl)-N $N^{4}$-(7-chloro-3-fluoroquinolin-4-yl)pentane-1,4-diamine & FClAQ6 & 32 \\
\hline & $N^{1}$-[2-(1-adamantyl)ethyl]- $N^{4}$-(7-chloro-3-fluoroquinolin-4-yl)pentane-1,4-diamine & FClAQ7 & 45 \\
\hline & $N^{\prime}$-(7-chloro-3-fluoroquinolin-4-yl)- $N, N$-diethylpropane-1,3-diamine & FClAQ8 & 73 \\
\hline \multirow[t]{7}{*}{$\mathrm{FCl} 2 \mathrm{AQ}$} & $N^{1}$-(1-adamantylmethyl)- $N^{2}$-(7-chloro-3-fluoroquinolin-2-yl)propane-1,2-diamine & FCl2AQ1 & 68 \\
\hline & $N^{1}$-(1-adamantylmethyl)- $N^{3}$-(7-chloro-3-fluoroquinolin-2-yl)butane-1,3-diamine & $\mathrm{FCl} 2 \mathrm{AQ} 2$ & 69 \\
\hline & $N^{1}$-[2-(1-adamantyl)ethyl]-N33-(7-chloro-3-fluoroquinolin-2-yl)butane-1,3-diamine & $\mathrm{FCl} 2 \mathrm{AQ} 3$ & 71 \\
\hline & $N^{1}$-(1-adamantylmethyl)- $N^{4}$-(7-chloro-3-fluoroquinolin-2-yl)pentane-1,4-diamine & $\mathrm{FCl} 2 \mathrm{AQ} 4$ & 70 \\
\hline & $N^{1}$-[2-(1-adamantyl)ethyl]- $N^{4}$-(7-chloro-3-fluoroquinolin-2-yl)pentane-1,4-diamine & FCl2AQ5 & 72 \\
\hline & $N^{4}$-(7-chloro-3-fluoroquinolin-2-yl)- $N^{1}, N^{1}$-diethylpentane-1,4-diamine & FCl2AQ6 & 76 \\
\hline & $N^{\prime}$-(7-chloro-3-fluoroquinolin-2-yl)- $N, N$-diethylpropane-1,3-diamine & $\mathrm{FCl} 2 \mathrm{AQ} 7$ & 75 \\
\hline
\end{tabular}

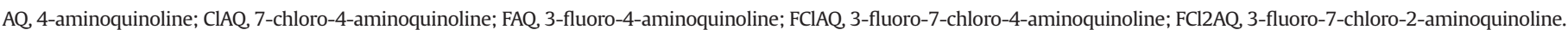

$[6,7]$. Mutations in the P. falciparum CQ resistance transporter (PfCRT) gene have a central role in CQ resistance. PfCRT is located in the FV membrane and, when mutated, increases CQ export from the FV and decreases its concentration inside the parasite $[6,8,9]$.

The aminoquinoline structure is very well known as a moiety useful for the design and development of new antimalarial agents [10-14]. Synthetic quinoline derivatives remain the most promising basis for discovery of new drugs [15], especially if they are effective against strains of Plasmodium resistant to CQ [16,17], and 4-aminoquinoline derivatives continue to be the most sought after antimalarial agents for chemical modification [18]. Efforts to develop new aminoquinolines include overcoming CQ resistance by adding modifications at the ring or at the side chain, with the main aim of finding new compounds that are not recognised by mutant transporters and thus cannot be pumped out of the parasite FV.

Recently, the synthesis of a series of aminoquinolines and tetraoxanes with demonstrated antiplasmodial activity, including activity both against the liver and blood stages, has been described [19]. Here we report on further examination of the aminoquinoline series in different in vitro model systems and provide further evidence for the complete curative effect observed in vivo by two compounds despite, at least in one case, a poor in vitro effect.

\section{Materials and methods}

\subsection{Parasites}

Cultures of a chloroquine-sensitive $\left(\mathrm{CQ}^{\mathrm{S}}\right)$ 3D7 and a chloroquineresistant $\left(\mathrm{CQ}^{\mathrm{R}}\right) \mathrm{Dd} 2$ strain of $P$. falciparum were maintained in human erythrocytes as described previously [20]. For in vitro drug assays, parasites were synchronised with $5 \%$ sorbitol and ring-stage parasites were then seeded in 96 -well plates to achieve $2 \%$ parasitaemia and $0.75 \%$ haematocrit.

In vivo testing was performed using the Plasmodium berghei ANKA strain maintained through serial intraperitoneal passage in C57BL/6 mice.

\subsection{Mice}

Female C57BL/6 mice (Medical Military Academy Animal Research Facility, Belgrade, Serbia), weighing 19-21 g, were used. Groups of four to six animals were housed in the Institute for Medical Research Animal Facility of the University of Belgrade (Belgrade, Serbia) under a natural photoperiod and were offered drinking water and standard feed ad libitum.

\subsection{Compounds}

A total of 26 experimental aminoquinoline derivatives with adamantane or benzothiophene as a carrier, synthesised at the Faculty of Chemistry of the University of Belgrade, were examined (Table 1).

According to the modifications at the aminoquinoline moiety structure, the compounds belonged to five groups as follows: (i) 4-aminoquinoline (AQ) ( $n=3$ compounds); (ii) 7-chloro-4aminoquinoline (ClAQ) $(n=6)$; (iii) 3-fluoro-4-aminoquinoline (FAQ) $(n=2)$; (iv) 3-fluoro-7-chloro-4-aminoquinoline (FClAQ) $(n=8)$; and (v) 3-fluoro-7-chloro-2-aminoquinoline (FCl2AQ) $(n=7)$.

For experimental use in vitro, the compounds were dissolved in dimethyl sulfoxide (DMSO) at a stock concentration of $50 \mathrm{mM}$. Compounds were further diluted in complete RPMI 1640 culture medium so that the final DMSO concentration was $\leq 0.2 \%$.

Compounds further investigated in vivo were suspended in $0.5 \%$ hydroxyethyl cellulose/0.1\% Tween 80 and were administered per os (p.o.).

\subsection{Experimental design}

All compounds were screened in vitro by the lactate dehydrogenase (LDH) assay adapted for Plasmodium [21], and those that at a defined concentration inhibited proliferation of either Plasmodium strain by $\geq 50 \%$ were titrated to obtain $50 \%$ inhibitory concentration $\left(\mathrm{IC}_{50}\right)$ values and were examined for in vivo efficacy. 
Prior to in vivo examination, compound toxicity was examined by treating uninfected mice with $160 \mathrm{mg} / \mathrm{kg} /$ day (the highest administered dose) of each compound for three consecutive days. A drug was considered non-toxic if mice did not develop any gross clinical symptoms (ruffled fur, lethargy or weight loss) during a 30day observation period. Compounds determined to be non-toxic were evaluated for antimalarial efficacy at doses of $80 \mathrm{mg} / \mathrm{kg} /$ day and $160 \mathrm{mg} / \mathrm{kg} / \mathrm{day}$. Compound efficacy was evaluated based on parasitaemia over time and survival of treated versus untreated mice. Cure was defined as survival past Day 31 post-infection (p.i.) and complete clearance of parasitaemia. Survival past Day 31 p.i. with residual parasitaemia indicated survival without cure. If a compound did not afford survival but significantly prolonged time to death of treated versus untreated mice $(P<0.05)$, the effect was defined as prolonged survival. Finally, if a compound cured mice at a dose of $80 \mathrm{mg} / \mathrm{kg} /$ day, efficacy was tested at lower doses, including 40, 20 and $10 \mathrm{mg} / \mathrm{kg} /$ day. Parasitaemia was determined twice weekly starting from Day 3 p.i. (immediately before treatment) and only mice in which parasitaemia was detected were submitted to experimental treatment. Parasitaemia was evaluated by microscopic examination of Giemsa-stained thin blood smears prepared from mouse tail blood on a Zeiss Axioscope 2+ optical microscope at 1000x magnification, whilst parasite clearance was additionally confirmed in treated survivors by quantitative PCR (qPCR).

\subsection{In vitro examination of compound efficacy}

In vitro testing was performed using a LDH assay. Compounds were first screened at a concentration of $500 \mathrm{nM}$ and those that showed a minimum of $50 \%$ growth inhibition of parasites of either strain (3D7 or Dd2) were further examined to obtain the $\mathrm{IC}_{50}$ value. Three independent experiments were performed for each compound, each with three replicates per condition. The assay was performed in flat-bottom 96-well microtitre plates. Briefly, compounds were tested at eight different concentrations ranging from $2 \mathrm{nM}$ to $256 \mathrm{nM}$, plated in a volume of $100 \mu \mathrm{L}$. Parasites were plated into the wells while in the ring phase at $0.75 \%$ haematocrit and $2 \%$ parasitaemia in a volume of $100 \mu \mathrm{L}$. Each well contained the compound and parasite culture in a final volume of $200 \mu \mathrm{L}$. Following incubation at $37^{\circ} \mathrm{C}$ for $48 \mathrm{~h}$ in a Heracell ${ }^{\mathrm{TM}} 150 \mathrm{i}$ incubator (Thermo Scientific, Waltham, MA), parasites were harvested and were subjected to three 20-min freeze-thaw cycles to resuspend the culture. Cultured erythrocytes without drug were used as the assay blank, whilst infected erythrocytes without drug were used as the assay control. CQ was used as the positive control for drug efficacy. To initiate the $\mathrm{LDH}$ reaction, $120 \mu \mathrm{L}$ of the detection reagent mixture (Malstat and NBT/PES) was aliquoted into a new flat-bottom 96well microtitre plate to which a $20 \mu \mathrm{L}$ sample of each parasite culture was added. Colour development of the LDH plate was detected using a Multiskan ${ }^{\circledR} \mathrm{X}$ microplate reader (Thermo Scientific) at $620 \mathrm{~nm}$ following $1 \mathrm{~h}$ of incubation in the dark. All reagents used in the assay were purchased from Sigma-Aldrich Inc. (St Louis, MO).

\subsection{In vivo examination of compound efficacy}

Antimalarial activity in vivo was tested by the modified Thompson test [22]. Infected erythrocytes were obtained from the peripheral blood of a donor mouse infected with $P$. berghei. Mice were inoculated intraperitoneally with $10^{6}$ infected erythrocytes diluted in phosphate-buffered saline (PBS) to a total volume of $250 \mu \mathrm{L}$ (Day 0 ). Mice were treated with the investigational compounds once a day for three consecutive days (Days 3, 4 and 5 p.i.). All compounds were administered p.o. at doses ranging from $10 \mathrm{mg} / \mathrm{kg} /$ day to $160 \mathrm{mg} / \mathrm{kg} /$ day in a total volume of $200 \mu \mathrm{L}$. Survival and parasitaemia were monitored for 30 days p.i. Parasitaemia was evaluated by microscopic examination of thin blood smears.

\section{7. $P C R$}

Residual parasitaemia was examined in the surviving mice by the real-time qPCR method adapted from Rougemont et al based on detection of the Plasmodium species-specific 18S rRNA gene [23]. Briefly, mice alive past day 31 p.i. and with complete parasite clearance were sacrificed and blood $(300-500 \mu \mathrm{L})$ was sampled from the left ventricle of the heart. The liver was removed, was rinsed with Dulbecco's PBS and was homogenised. DNA extraction was performed using $100 \mu \mathrm{L}$ of blood and liver homogenate samples using a DNeasy ${ }^{\circledR}$ Blood and Tissue Kit (QIAGEN, Hilden, Germany) according to the manufacturer's instructions. Each PCR reaction contained $1 \times$ Maxima $^{\mathrm{TM}}$ Probe qPCR Master Mix (Thermo Fisher Scientific), $200 \mathrm{nM}$ of each primer, $50 \mathrm{nM}$ probe, $1 \mathrm{U}$ of UNG (Thermo Fisher Scientific) and $3 \mu \mathrm{L}$ of template DNA in a final volume of $20 \mu \mathrm{L}$. PCR conditions were as follows: one holding step at $50^{\circ} \mathrm{C}$ for $2 \mathrm{~min}$; one holding step at $95^{\circ} \mathrm{C}$ for $10 \mathrm{~min}$; followed by 45 cycles of $95^{\circ} \mathrm{C}$ for $15 \mathrm{~s}$ and $60^{\circ} \mathrm{C}$ for $1 \mathrm{~min}$. Samples with $\mathrm{C}_{\mathrm{t}}$ values $>40$ were considered negative. A positive ( $P$. berghei DNA) and a negative $\left(\mathrm{H}_{2} \mathrm{O}\right)$ control were included in each run.

\subsection{Statistical analysis}

$\mathrm{IC}_{50}$ values were obtained using a sigmoidal dose-response model with the variable slope fitted to the results. Survival rates in each particular group were estimated by the Kaplan-Meier product limit method and were compared by the log-rank (two curves) and logrank for trends (three or more curves) tests. The level of statistical significance was 0.05 . Statistical analysis was performed using GraphPad Prism v.5 (GraphPad Software Inc., La Jolla, CA).

\section{Results}

A series of 26 aminoquinolines was examined in this work. Of these, 12 compounds inhibited proliferation of either the $\mathrm{CQ}^{\mathrm{S}}$ or $\mathrm{CQ}^{\mathrm{R}}$ Plasmodium strain by $\geq 50 \%$; the remaining 14 compounds did not and thus were eliminated from further study.

All 12 compounds were first assayed for toxicity. Four compounds ( $\mathrm{AQ} 2, \mathrm{AQ} 3, \mathrm{ClAQ} 3$ and $\mathrm{ClAQ6}$ ) were shown to cause acute toxicity at a dose of $160 \mathrm{mg} / \mathrm{kg} / \mathrm{day}$, three of which were eliminated from further in vivo examination. However, due to chemical similarity with other members of the benzothiophene group, which were non-toxic even at the highest applied dose, one of the latter compounds (ClAQ3), although toxic at $160 \mathrm{mg} / \mathrm{kg} / \mathrm{day}$, was further tested for toxicity at $80 \mathrm{mg} / \mathrm{kg} /$ day and was found to be non-toxic at this dose. ClAQ3 was thus included in the in vivo examination (Table 2).

A total of nine compounds (AQ1, ClAQ1, ClAQ2, ClAQ3, ClAQ4, ClAQ5, FAQ1, FClAQ1 and FClAQ2) were subjected to in vivo testing. When administered at doses of $80 \mathrm{mg} / \mathrm{kg} /$ day and/or $160 \mathrm{mg} / \mathrm{kg} /$ day, all nine compounds significantly prolonged survival of treated versus untreated mice $(P<0.05$; Figs 1 and 2$)$.

Remarkably, three ClAQ compounds (ClAQ1, ClAQ4 and ClAQ5) and one FClAQ compound (FClAQ1) (chemical structures presented in Table 3 ) afforded survival of $60-100 \%$ of treated mice past Day 31. Of these, ClAQ4 and ClAQ5 afforded a 60-80\% survival rate of infected mice, although not even the highest dose of either compound eradicated parasitaemia in a single animal.

However, treatment with $80 \mathrm{mg} / \mathrm{kg} /$ day and $160 \mathrm{mg} / \mathrm{kg} /$ day of the other two compounds (ClAQ1 and FClAQ1) afforded complete cure. All treated infected mice survived beyond Day 31 (Figs 3 and 4) and, moreover, survival was associated with parasite clearance as determined by microscopic examination and by qPCR of murine blood and liver tissues after Day 31. Thus, we next examined their effect at lower doses, which revealed a strong dose-dependent effect ( $P=0.0141$ and $P=0.0362$ for ClAQ1 and FClAQ1, respectively), but 
Table 2

Antimalarial effect of experimental aminoquinolines examined in vitro and in vivo.

\begin{tabular}{|c|c|c|c|c|c|c|}
\hline \multirow[t]{2}{*}{ Group } & \multirow[t]{2}{*}{ Compound } & \multicolumn{2}{|c|}{$\begin{array}{l}\text { In vitro LDH assay } \\
\left.\text { [GM IC }{ }_{50} \text { value }(\mathrm{nM})\right]\end{array}$} & \multicolumn{3}{|l|}{ In vivo Thompson test } \\
\hline & & Strain $3 \mathrm{D} 7^{\mathrm{a}}$ & Strain Dd ${ }^{b}$ & Toxicity at $160 \mathrm{mg} / \mathrm{kg} /$ day & Treatment dose (mg/kg/day) & Effect on Day 31 p.i. \\
\hline \multirow[t]{3}{*}{$\mathrm{AQ}$} & AQ1 & 14.08 & 118.2 & NT & 80 & Prolonged time to death $*(P=0.0031)$ \\
\hline & AQ2 & 99.86 & 195.3 & $\mathrm{~T}$ & & \\
\hline & AQ3 & 67.33 & 223.0 & $\mathrm{~T}$ & & \\
\hline \multirow[t]{8}{*}{$\mathrm{ClAQ}$} & ClAQ1 & 34.75 & 58.4 & NT & 160,80 & $100 \%$ cure \\
\hline & & & & & $40,20,10$ & Prolonged time to death * $(P=0.0031,0.0067,0.0031)$ \\
\hline & ClAQ2 & 142.70 & $>500$ & NT & 80 & Prolonged time to death * $(P=0.0031)$ \\
\hline & ClAQ3 & 43.48 & 34.8 & $\mathrm{~T}$ & 80 & Prolonged time to death $*(P=0.0067)$ \\
\hline & ClAQ4 & 32.53 & 13.7 & NT & 160 & $75 \%$ survival ${ }^{\mathrm{c}}(P=0.0067)$ \\
\hline & & & & & 80 & $80 \%$ survival ${ }^{\mathrm{c}}(P=0.0031)$ \\
\hline & ClAQ5 & 34.13 & 16.7 & NT & 160 & $60 \%$ survival ${ }^{\mathrm{c}}(P=0.002)$ \\
\hline & ClAQ6 & 67.07 & 35.9 & $\mathrm{~T}$ & & \\
\hline FAQ & FAQ1 & 185.38 & $>500$ & NT & 80 & Prolonged time to death $*(P=0.0290)$ \\
\hline \multirow[t]{4}{*}{ FClAQ } & FClAQ1 & 41.13 & $>500$ & NT & 160,80 & $100 \%$ cure \\
\hline & & & & & 40 & Prolonged time to death $*(P=0.0031)$ \\
\hline & & & & & 20,10 & $\mathrm{~N} / \mathrm{S}(P>0.05)$ \\
\hline & FClAQ2 & 145.36 & $>500$ & NT & 160 & Prolonged time to death $*(P=0.0020)$ \\
\hline Control & CQ & 18.74 & 249.1 & NT & 160 & $100 \%$ cure \\
\hline
\end{tabular}

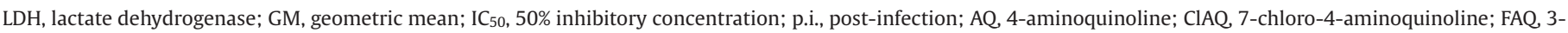
fluoro-4-aminoquinoline; FClAQ, 3-fluoro-7-chloro-4-aminoquinoline; CQ, chloroquine; NT, non-toxic; T, toxic; N/S, not significant.

* Versus infected untreated (control) mice.

a CQ-sensitive Plasmodium falciparum strain 3D7.

b CQ-resistant $P$. falciparum strain Dd2.

c With residual parasitaemia.

did not afford survival. ClAQ1 is particularly interesting in this respect as treatment with even the lowest dose $(10 \mathrm{mg} / \mathrm{kg})$ prolonged survival $(P=0.0031)$. However, dose reduction resulted in persistence of parasitaemia in all mice.

On the other hand, an interesting observation with FClAQ1 was that although all mice treated with $40 \mathrm{mg} / \mathrm{kg} /$ day eventually succumbed to the infection, they were able to tolerate very high levels of parasitaemia, which amounted up to $62 \%$ (range $37.5-62.4 \%$ ). In contrast, the highest level of parasitaemia observed with any other treatment regimen ranged from as low as $0.1 \%$ to not more than $13.9 \%$ (Table 4 ).

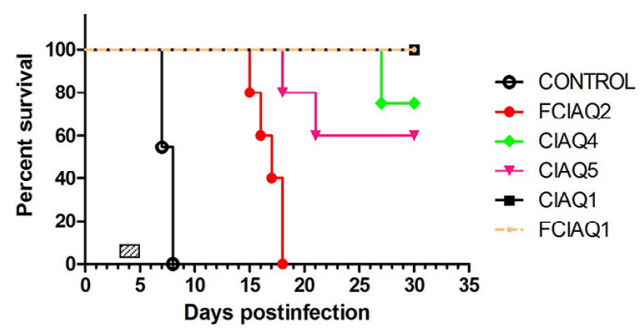

Fig. 1. Effect of a 3-day treatment with $160 \mathrm{mg} / \mathrm{kg} / \mathrm{day}$ of the investigational compounds on the survival of mice infected with Plasmodium berghei ANKA strain. $\square$, treatment days.

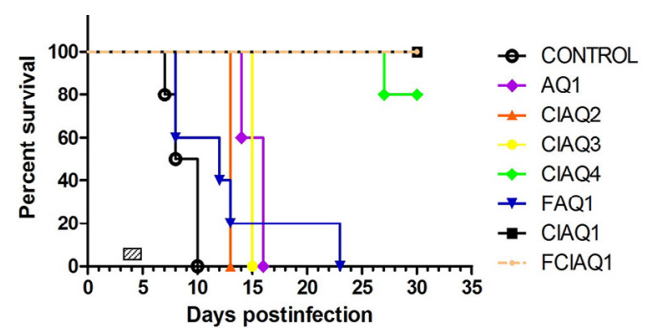

Fig. 2. Effect of a 3-day treatment with $80 \mathrm{mg} / \mathrm{kg} /$ day of the investigational compounds on the survival of mice infected with Plasmodium berghei ANKA strain. $\square$, treatment days.
Interestingly, the correlation between the in vivo and in vitro results appeared indiscriminate (Table 2 ). The four compounds with the highest in vivo efficacy did not show the best in vitro results, i.e. the lowest $\mathrm{IC}_{50}$ values for both strains. For instance, ClAQ4 and ClAQ5, the two compounds that afforded survival but not cure, had quite low $\mathrm{IC}_{50}$ values and by far the lowest ones against the $\mathrm{CQ}^{\mathrm{R}}$ strain. In contrast, FClAQ1, which cured all infected mice (in two doses), had no in vitro effect against the $\mathrm{CQ}^{\mathrm{R}}$ strain $(>500 \mathrm{nM})$. On the other hand, AQ1, the single compound that had lower $\mathrm{IC}_{50}$ values than $\mathrm{CQ}$ against both strains, did not have remarkable in vivo efficacy. Of the remaining four compounds, which all significantly prolonged the

Table 3

Chemical structures of the most active investigational compounds in vivo.<smiles>CC(CCNCC12CC3CC(CC(C3)C1)C2)Nc1ccnc2cc(Cl)ccc12</smiles> 


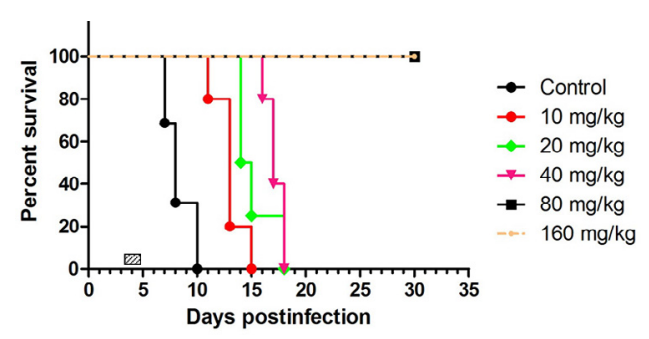

Fig. 3. Effect of a 3-day treatment with $\mathrm{ClAQ} 1$ in the full dosage regimen on the survival of mice infected with Plasmodium berghei ANKA strain. $\mathbb{Z}$, treatment days.

survival time of treated infected mice, three had much higher $\mathrm{IC}_{50}$ values than CQ against both parasite strains (Table 2).

\section{Discussion}

Here we present the antimalarial efficacy of a series of investigational aminoquinoline compounds. Of the 12 compounds that exhibited $\geq 50 \%$ growth inhibition in parasite cultures, the efficacy of nine compounds shown to be non-toxic in vivo was examined in a mouse infection model. When given in three daily doses of $80 \mathrm{mg} / \mathrm{kg}$ or $160 \mathrm{mg} / \mathrm{kg}$, all nine compounds significantly prolonged survival compared with untreated controls, but most notably, four compounds afforded survival of mice past Day 31. Of these, compounds ClAQ4 and ClAQ5 afforded a high protection rate although with residual infection in mice that survived the observation period, whilst compounds ClAQ1 and FClAQ1 afforded cure (with parasite clearance) for $100 \%$ of mice at doses both of $160 \mathrm{mg} / \mathrm{kg} /$ day and $80 \mathrm{mg} / \mathrm{kg} /$ day. At the latter dose, the survival rate afforded by ClAQ1 and FClAQ1 was even superior to that of CQ. Furthermore, these two compounds showed significant activity at lower doses as well, of which ClAQ1 prolonged time to death (versus untreated controls) even at a dose as low as $10 \mathrm{mg} / \mathrm{kg}$.

Several important observations arise from these data. First, we have observed that the best in vivo effects did not correlate with in vitro efficacy. For instance, AQ1 was the single compound that had lower $\mathrm{IC}_{50}$ values than CQ against both strains, but its in vivo efficacy did not go beyond prolonging survival of infected treated mice. On the other hand, none of the three examined compounds with fluorine on the aminoquinoline moiety had any effect of against the $\mathrm{CQ}^{\mathrm{R}}$ strain in vitro, yet all significantly prolonged survival of infected treated mice, whilst FClAQ1 even afforded complete cure. Such discordance has been previously reported for some thiophene- and furan-based aminoquinolines synthesised by the same group [24]. The discrepancy between in vitro and in vivo effects suggests that the antimalarial efficacy of such compounds is due to their metabolites rather than the compounds themselves.

The second interesting observation was that although FClAQ1 at lower doses did not afford survival, it allowed mice to survive

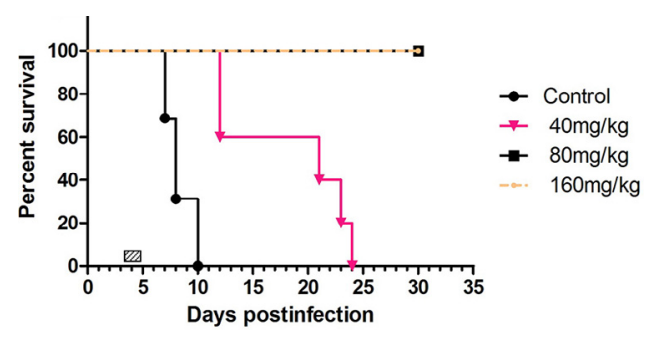

Fig. 4. Effect of a 3-day treatment with FClAQ1 in three dosage regimens (40, 80 and $160 \mathrm{mg} / \mathrm{kg}$ ) on the survival of mice infected with Plasmodium berghei ANKA strain. 范 treatment days.

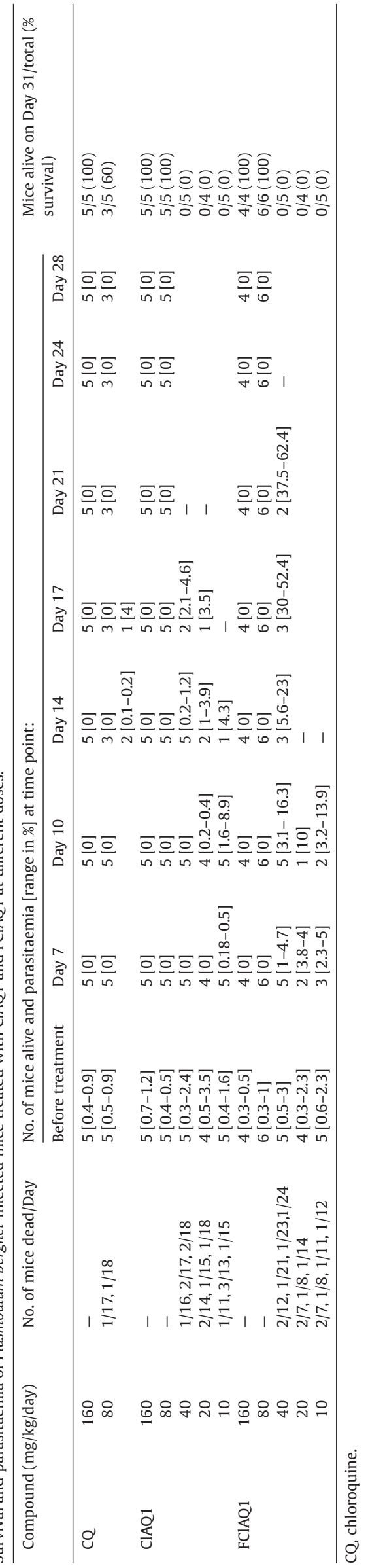


remarkably high parasite burdens (37-62\%), as opposed to the highest parasitaemia of only $14 \%$ appearing to be the survival limit by any other treatment. Importantly, this compound (designated compound 25 in [19]) has been shown to have significant activity in the plasmodial liver stage infection [19], where the intrahepatocytic inhibition of parasite growth was attributed to the presence of the fluorine atom at the $C(3)$ position on the aminoquinoline moiety. The ability of mice treated with this compound to survive massive parasitaemia may indicate its impact on parasite pathogenicity/virulence.

Importantly, the approach taken in this study, i.e. to examine all compounds that exerted $\geq 50 \%$ parasite growth inhibition in vitro in parallel with their effects in an in vivo infection model, allowed us to observe a therapeutic potential that would have gone unnoticed had we chosen the usual approach to examine in vivo only those compounds with an $\mathrm{IC}_{50}$ lower than that of the control drug. This observation also suggests that there may have been drug candidates in the past that have been missed because of the approach. It is to be hoped that highly advanced techniques including highthroughput technologies will help avoid such unfortunate events in the future.

A look at the chemical structures of the four most effective compounds (Table 3 ) shows that the carrier in ClAQ4 and ClAQ5 is benzothiophene, whilst in the case of ClAQ1 and FClAQ1 it is adamantane. Since the results showed that both compounds with adamantane afforded cure of mice, it appears that the higher in vivo activity may be attributed to its use as a carrier. Among its many biological properties, adamantane has been shown to substantially increase drug solubility in lipophilic membranes and may thus increase compound uptake [25].

In summary, the results presented here illustrate the enormous potential of aminoquinoline derivatives bearing an adamantane group as antimalarials whose metabolites and mechanisms of action warrant further investigation and put adamantane into the spotlight as a carrier that enhances the antimalarial effect of aminoquinolines.

\section{Acknowledgments}

JS was a recipient of the Young Scientist Award for a presentation based on these results at the 12th European Multicolloquium of Parasitology, 20-24 July 2016, Turku, Finland.

Funding: This work was supported by grants from the Ministry of Education, Science and Technological Development of the Republic of Serbia [nos. III 41019 and ON172008].

Competing interests: None declared.

Ethical approval: This study was carried out in accordance with the ARRIVE guidelines and was approved by the Veterinary Directorate of the Ministry of Agriculture and Environmental Protection of Serbia [decision no. 323-07-02444/2014-05/1].

\section{References}

[1] White NJ, Pukrittayakamee S, Hien TT, Faiz MA, Mokuolu OA, Dondorp AM. Malaria. Lancet 2014;383:723-35.
[2] World Health Organization. World malaria report 2016. Geneva, Switzerland: WHO; 2016 http://www.who.int/malaria/media/world-malaria-report-2016/en/. [Accessed 7 March 2017].

[3] Tatem AJ, Jia P, Ordanovich D, Falkner M, Huang Z, Howes R, et al. The geography of imported malaria to non-endemic countries: a meta-analysis of nationally reported statistics. Lancet Infect Dis 2017;17:98-107.

[4] Fidock DA, Rosenthal PJ, Croft SL, Brun R, Nwaka S. Antimalarial drug discovery: efficacy models for compound screening. Nat Rev Drug Discov 2004;3:50920.

[5] Aguiar ACC, da Rocha EMM, de Souza NB, França TCC, Krettli AU. New approaches in antimalarial drug discovery and development-a review. Mem Inst Oswaldo Cruz 2012;107:831-45.

[6] Pulcini S, Staines HM, Lee AH, Shafik SH, Bouyer G, Moore CM, et al. Mutations in the Plasmodium falciparum chloroquine resistance transporter, PfCRT, enlarge the parasite's food vacuole and alter drug sensitivities. Sci Rep 2015;5:14552.

[7] Combrinck JM, Mabotha TE, Ncokazi KK, Ambele MA, Taylor D, Smith PJ, et al. Insights into the role of heme in the mechanism of action of antimalarials. ACS Chem Biol 2013;8:133-7.

[8] Wellems TE, Plowe CV. Chloroquine-resistant malaria. J Infect Dis 2001;184:770-6.

[9] Gadalla NB, Malmberg M, Adam I, Oguike MC, Beshir K, Elzaki SE, et al. Alternatively spliced transcripts and novel pseudogenes of the Plasmodium falciparum resistance-associated locus pfcrt detected in East African malaria patients. J Antimicrob Chemother 2015;70:116-23.

[10] Šolaja BA, Opsenica D, Smith KS, Milhous WK, Terzić N, Opsenica I, et al. Novel 4-aminoquinolines active against chloroquine-resistant and sensitive $P$. falciparum strains that also inhibit botulinum serotype A. J Med Chem 2008;51:4388-91.

[11] Casagrande M, Barteselli A, Basilico N, Parapini S, Taramelli D, Sparatore A. Synthesis and antiplasmodial activity of new heteroaryl derivatives of 7-chloro4-aminoquinoline. Bioorg Med Chem 2012;20:5965-79.

[12] Manohar S, Rajesh UC, Khan SI, Tekwani BL, Rawat DS. Novel 4-aminoquinoline-pyrimidine based hybrids with improved in vitro and in vivo antimalarial activity. ACS Med Chem Lett 2012;3:555-9.

[13] Sáenz FE, Mutka T, Udenze K, Oduola AMJ, Kyle DE. Novel 4-aminoquinoline analogs highly active against the blood and sexual stages of Plasmodium in vivo and in vitro. Antimicrob Agents Chemother 2012;56:4685-92.

[14] Tukulula M, Njoroge M, Abay ET, Mugumbate GC, Wiesner L, Taylor D, et al. Synthesis and in vitro and in vivo pharmacological evaluation of new 4-aminoquinoline-based compounds. ACS Med Chem Lett 2013;4:1198-202.

[15] Kaur K, Jain M, Reddy RP, Jain R. Quinolines and structurally related heterocycles as antimalarials. Eur J Med Chem 2010;45:3245-64.

[16] Opsenica I, Burnett JC, Gussio R, Opsenica D, Todorović N, Lanteri CA, et al. A chemotype that inhibits three unrelated pathogenic targets: the botulinum neurotoxin serotype A light chain, P. falciparum malaria, and the Ebola filovirus. J Med Chem 2011;54:1157-69.

[17] Opsenica IM, Tot M, Gomba L, Nuss JE, Sciotti RJ, Bavari S, et al. 4-Amino-7chloroquinolines: probing ligand efficiency provides botulinum neurotoxin serotype A light chain inhibitors with significant antiprotozoal activity. J Med Chem 2013;56:5860-71.

[18] O'Neill PM, Bray PG, Hawley SR, Ward SA, Park KB. 4-Aminoquinolines-past, present, and future: a chemical perspective. Pharmacol Ther 1998;77:29-58.

[19] Terzić N, Konstantinović J, Tot M, Burojević J, Djurković-Djaković O, Srbljanović J, et al. Reinvestigating old pharmacophores: are 4-aminoquinolines and tetraoxanes potential two-stage antimalarials? J Med Chem 2016;59:264-81.

[20] Trager W, Jensen JB. Human malaria parasites in continuous culture. Science 1976;193:673-5.

[21] Makler MT, Ries JM, Williams JA, Bancroft JE, Piper RC, Gibbins BL, et al. Parasite lactate-dehydrogenase as an assay for Plasmodium falciparum drug-sensitivity. Am J Trop Med Hyg 1993;48:739-41.

[22] Ager AL. Experimental models: rodent malaria models (in vivo). In: Peters W, Richards WHG, editors. Handbook of experimental pharmacology: antimalarial drugs. New York, NY: Springer Verlag; 1983. p. 225-54.

[23] Rougemont M, Van Saanen M, Sahli R, Hinrikson HP, Bille J, Jaton K. Detection of four Plasmodium species in blood from humans by 18S rRNA gene subunitbased and species-specific real-time PCR assays. J Clin Microbiol 2004;42:563643.

[24] Opsenica IM, Verbić TŽ, Tot M, Sciotti RJ, Pybus BS, Djurković-Djaković O, et al. Investigation into novel thiophene- and furan-based 4-amino-7chloroquinolines afforded antimalarials that cure mice. Bioorg Med Chem 2015;23:2176-86.

[25] Mans GA. Diamondoid molecules. Adv Chem Phys 2007;136:207-58. 